\title{
均三溙衍生物的合成、结构及二阶非线性 光学性质
}

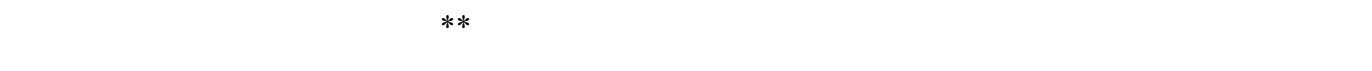

(1) 山东大学晶体材料国家重点实验室, 济南 250100; (2) 山东轻工业学院化工系, 济南 250100; (3)山东大学信息科学与工程学院，济南 250100; (4)华中科技大学生命科学与技术学院，武汉 430074)

摘要 合成了三个均三嗪衍生物并测定了相应的晶体结构. 化合物 I: 2,4-Dimethyl-6- $(p-N$, $N$-dimethylaminostyryl)-s-triazine (简称为NMe-1). 化合物 II: 2,4-Dimethyl-6-( $p$ - $N, N$-diethylaminostyryl)-s-triazine (简称为NEt-1). 化合物III: 2-Methyl-4,6-bis ( $p$ - $N, N$-dimethylaminostyryl)-s-triazine (简 称为NMe-2). NMe-1 和NEt-1 属D- $\pi$-A型一维电荷转移化合物, 前者结晶为中心对称的空间群 $\mathrm{P} \overline{1}$; 后者结晶为极性空间群 $\mathrm{Cc}$, 分子电荷转移轴同向平行排列. 在 $1064 \mathrm{~nm}$ 基频光下, NEt-1 的粉 晶倍频光强度为尿素的 46.8 倍. NMe- 2 具有 $\Lambda$ 型二维电荷转移结构, 结晶为极性空间群 $\mathrm{P} 2{ }_{1}$, 其粉 晶倍频光强度为尿素的 46.2 倍. 用量化计算和张量分析的方法探讨了分子的微观二阶非线性系 数 $(\beta$ 值)和堆积方式对晶体的宏观二阶非线性效应的影响.

\section{关键词 均三嗪衍生物 合成 晶体结构 二次谐波产生(SHG) 分子二阶极化率 $(\beta)$}

有机非线性光学(Nonlinear Optics, NLO)也称分 子非线性光学. 近 30 余年来, 有机NLO材料由于具 有大的NLO系数和结构多样性而得到广泛研究 ${ }^{[1 \sim 3]}$. 二次谐波产生(Second harmonic generation, $\mathrm{SHG}$ )是二 阶 NLO 过程，由分子的微观二阶极化率 $\beta$ 和晶体的 宏观二阶极化率 $\chi^{(2)}$ 表征. 文献上 SHG 强度多指宏观 倍频强度, 与 $\chi^{(2)}$ 的平方成正比. 对于有机晶体, 影 响SHG的因素可归结为: (1) 分子的二阶极化率 $\beta$. 具 有大 $\beta$ 值的 NLO 化合物大多为 D- $\pi$-A 型, 即在 $\pi$-共轭
体系的两端分别连有电子给体 $\mathrm{D}$ 和受体 $\mathrm{A}^{[4-9]}$. (2)晶体 中分子的堆积方式. 分子的 $\beta$ 和晶体的 $\chi^{(2)}$ 均为三阶 张量, 分子非中心对称且相应晶体也非中心对称才 可能具有宏观 SHG 效应. 由于偶极-偶极相互作用, 大多数偶极分子结晶为中心对称的结构. 人们提出 各种分子设计以克服中心对称结晶的倾向, 如采用 基态弱偶极、激发态强偶极的电荷转移分子 ${ }^{[10,11]}$, 手 性分子 ${ }^{[4]}, \Lambda$ 型分子 ${ }^{[12]}$ ，强氢键化合物 ${ }^{[7]}$, 以及非偶极 非中心对称的八极(octupolar)分子[13 15]. 由于有机分 
子结构的丰富性, 已有近 40 年研究历史的二阶分子 NLO 领域中, 仍不断涌现具有突出NLO功能的结构 新颖的化合物. 迄今为止, 大部分研究报告侧重于分 子的 $\beta$ 性质或侧重于晶体的SHG性质，而将微观与宏 观二阶NLO综合起来的研究相对较少 ${ }^{[1]}$.

本工作合成了三个均三嗪的衍生物(见图 1), 其中 NEt-1 为一维电荷转移分子, 其分子的电荷转移轴同 向平行排列, $\mathrm{NMe}-2$ 为 $\Lambda$ 型二维电荷转移分子, 这两 个化合物结晶为极性空间群, 都具有强的 SHG 效应.

\section{1 合成与结构}

\section{1 合成}

化合物 I: 2,4-Dimethyl-6-( $p-N, N$ - dimethylaminos-tyryl)-s-triazine( 缩写为 NMe-1). 三口瓶中, 加入 $3.7 \mathrm{~g}(0.03 \mathrm{~mol}) 2,4,6$-三甲基均三嗪、 $1.0 \mathrm{~g}$ 氢氧化钾和 $200 \mathrm{~mL}$ 甲醇. 溶解后, 在回流温度下将另一含有 2.23 $\mathrm{g}(0.015 \mathrm{~mol}) \mathrm{N}, \mathrm{N}$-二甲胺基苯甲醛的 $50 \mathrm{ml}$ 甲醇溶液 滴加到上述三口瓶中, $3 \mathrm{~h}$ 滴完. 保持回流 $24 \mathrm{~h}$. 用旋 转蒸发仪除去溶剂, 残余物用 $\mathrm{V}$ (苯)：V(乙醇) $=10$ : 1 的苯-乙醇混合物作为洗提剂, 通过硅胶柱色谱分 离得到黄色产品. 将产品溶于苯-乙醇混合溶剂, 室 温下缓慢挥发溶剂, 得到浅黄色块状晶体。产率: $60 \% \sim 65 \% . \mathrm{C}_{15} \mathrm{H}_{18} \mathrm{~N}_{4}$ 的计算值为 (\%): C, 70.84; H, 7.13; N, 22.03. 样品实测值为 $(\%)$ : C, 70.95; H, 7.05; $\mathrm{N}, 21.88$. 熔点: $147 \sim 149^{\circ} \mathrm{C} .{ }^{1} \mathrm{H}$ NMR $\left(\mathrm{CDCl}_{3}\right) \delta: 8.14$ $(\mathrm{d}, 1 \mathrm{H}, J=16.09 \mathrm{~Hz}), 7.53(\mathrm{~d}, 2 \mathrm{H}, J=8.78 \mathrm{~Hz}), 6.82(\mathrm{~d}$, $1 \mathrm{H}, J=16.09 \mathrm{~Hz}), 6.69(\mathrm{~d}, 2 \mathrm{H}, J=8.78 \mathrm{~Hz}), 3.03(\mathrm{~s}$, $6 \mathrm{H}), 2.60(\mathrm{~s}, 6 \mathrm{H})$.

化合物 II: 2,4-Dimethyl-6- $(p-N, N-\mathrm{d}$ iethylaminos-tyryl)-s-triazine(缩写为 NEt-1). 用 $N, N$-二乙胺基 苯甲醛替代 $N, N$-二甲胺基苯甲醛，用同样合成方法得 到黄色块状晶体，产率: $58 \% \sim 66 \% . \mathrm{C}_{17} \mathrm{H}_{22} \mathrm{~N}_{4}$ 的计算值 为 $(\%)$ : $\mathrm{C}, 72.31 ; \mathrm{H}, 7.85 ; \mathrm{N}, 19.84$. 样品实测值为 $(\%)$ :
C, 71.86; H, 7.96; N, 19.44. 熔点: 92 94 ${ }^{\circ} \mathrm{C} .{ }^{1} \mathrm{H}$ NMR $\left(\mathrm{CDCl}_{3}\right) \delta: 8.13(\mathrm{~d}, 1 \mathrm{H}, J=15.60 \mathrm{~Hz}), 7.50(\mathrm{~d}, 2 \mathrm{H}, J=$ $9.26 \mathrm{~Hz}), 6.80(\mathrm{~d}, 1 \mathrm{H}, J=15.60 \mathrm{~Hz}), 6.65(\mathrm{~d}, 2 \mathrm{H}, J=$ $9.26 \mathrm{~Hz}), 3.41(\mathrm{q}, 4 \mathrm{H}, J=6.99 \mathrm{~Hz}), 2.59(\mathrm{~s}, 6 \mathrm{H}), 1.20(\mathrm{t}$, $6 \mathrm{H}, 6.83 \mathrm{~Hz})$.

化合物 III: 2-Methyl-4,6-bis( $p$ - $N, N$ - dimethylaminostyryl)-s-triazine(缩写为 NMe-2): 合成步骤与化 合物I基本相同，但 $\mathrm{N}, \mathrm{N}-$ 二甲胺基苯甲醛与 2,4,6-三甲 基均三嗪的摩尔比调整为 $2.5: 1$, 最终得到黄绿色针 状晶体. 产率: $35 \% \sim 42 \% . \mathrm{C}_{24} \mathrm{H}_{27} \mathrm{~N}_{5}$ 的计算值为(\%): $\mathrm{C}$, $74.77 ; \mathrm{H}, 7.06 ; \mathrm{N}, 18.17$. 样品实测值为 $(\%)$ : C, 74.52; $\mathrm{H}, 6.97 ; \mathrm{N}, 17.94$. 熔点: $162 \sim 164^{\circ} \mathrm{C} .{ }^{1} \mathrm{H}$ NMR $\left(\mathrm{CDCl}_{3}\right)$ $\delta: 8.32(\mathrm{~d}, 2 \mathrm{H}, J=15.61 \mathrm{~Hz}), 7.71(\mathrm{~d}, 4 \mathrm{H}, J=9.26 \mathrm{~Hz})$, $7.04(\mathrm{~d}, 2 \mathrm{H}, J=15.60 \mathrm{~Hz}), 6.70(\mathrm{~d}, 4 \mathrm{H}, J=9.27 \mathrm{~Hz})$, $3.07(\mathrm{~s}, 12 \mathrm{H}), 2.73(\mathrm{~s}, 3 \mathrm{H})$.

熔点由 DSC 822e 型差示扫描量热仪测得, 核磁 共振谱由 FX-90Q 型谱仪测定, 紫外光谱由日立 U-3500 UV-VIS 光谱仪测得，元素分析用 PE-2400 自 动分析仪测定.

\section{2 晶体结构测定}

用 Bruker P4 四圆衍射仪，以 Mo $\mathrm{K} \alpha$ 射线 (经石 墨单色器)作为辐射源. $T=293 \mathrm{~K}$, 采用 $\omega-2 \theta$ 扫描方 式收集衍射点，并用半经验方法进行吸收校正。用 Shelex-97 程序进行结构解析. $\mathrm{X}$ 衍射数据及晶体学数 据列于表 1 .

\section{2 结果与讨论}

\section{1 晶体结构}

NMe-1 分子中三嗪环与苯环平面间的夹角仅为 $6.8^{\circ}$, 具有良好平面性，属 $\mathrm{D}-\pi-\mathrm{A}$ 型极性分子(见图 2). 其中二甲胺基作为电子给体, 三嗪环作为电子受体. 分 子平面与(010) 晶面平行, 构成层状堆积, 相邻两层分 子的电荷转移轴方向相反, 导致中心对称的晶体结构.


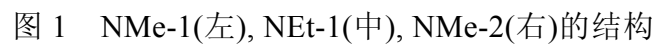


表 $1 \quad \mathrm{X}$ 衍射数据和晶体学数据

\begin{tabular}{|c|c|c|c|}
\hline 化合物 & NMe-1 & NEt-1 & NMe-2 \\
\hline 晶样尺度/ mm ${ }^{3}$ & $0.36 \times 0.32 \times 0.03$ & $0.42 \times 0.39 \times 0.34$ & $0.40 \times 0.22 \times 0.03$ \\
\hline 衍射点收集范围/(º) & $2.97 \leqslant \theta \leqslant 27.49$ & $2.11 \leqslant \theta \leqslant 27.50$ & $1.98 \leqslant \theta \leqslant 27.49$ \\
\hline 偏差因子 $[I>2 \sigma(I)]$ & $R=0.0775, w R=0.1951$ & $R=0.0450, w R=0.1079$ & $R=0.0636, w R=0.1050$ \\
\hline 衍射点, 独立衍射点 & 3898,3202 & 2229,2059 & 3775,3011 \\
\hline 晶系, 空间群 & 三斜, $\mathrm{P} \overline{1}$ & 单斜, Cc & 单斜, $\mathrm{P} 2{ }_{1}$ \\
\hline$a / \mathrm{nm}$ & $0.7834(5)$ & $1.55368(15)$ & $0.6528(5)$ \\
\hline$b / \mathrm{nm}$ & $0.7852(5)$ & $1.40904(13)$ & $0.7994(5)$ \\
\hline$c / \mathrm{nm}$ & $1.3105(5)$ & $0.86146(8)$ & $2.0615(5)$ \\
\hline$\alpha /\left(^{\circ}\right)$ & $81.090(5)$ & & \\
\hline$\beta /\left(^{\circ}\right)$ & $88.966(5)$ & $121.151(8)^{\circ}$ & $92.192(5)^{\circ}$ \\
\hline$\gamma /\left(^{\circ}\right)$ & $62.259(5)$ & & \\
\hline$D_{c} / \mathrm{g} \cdot \mathrm{cm}^{-3}$ & 1.201 & 1.162 & 1.191 \\
\hline$V / \mathrm{nm}^{3}$ & $0.7035(7)$ & $1.6140(3)$ & $1.0750(11)$ \\
\hline Z & 2 & 4 & 2 \\
\hline
\end{tabular}

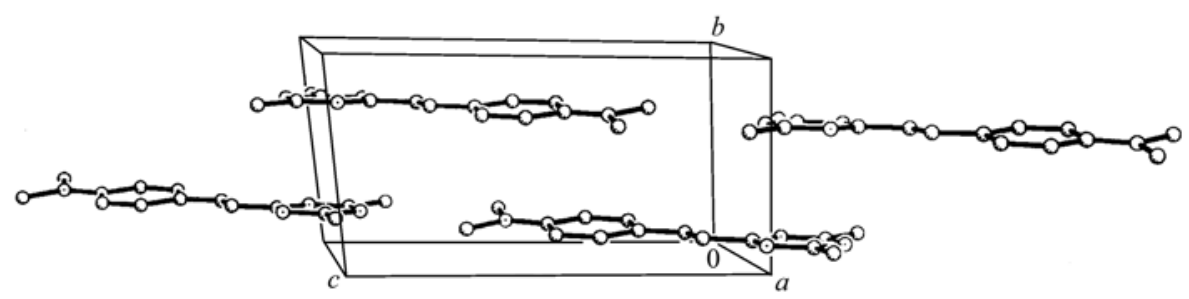

图 2 NMe-1 的晶体结构

NEt-1 晶体属于 $\mathrm{Cc}$ 空间群，晶胞中有 4 个分子. 参见图 1 和图 3, 定义胺基 $N$ 与三嗪环上的 $N^{\prime}$ 的连线 为电荷转移轴 (即分子坐标系中的 $z$ 轴), 则分子电荷 转移轴在晶体的宏观介电坐标系 $X Y Z$ (见图 3)下的方 向余弦为 $[-0.9999,-0.0071,-0.0047]$, 晶胞中其余 3 个分子的电荷转移轴也与 $X$ 轴 (即 $a$ 轴)一致, 这说 明晶体中所有分子的偶极矩方向完全相同.

$\mathrm{NMe}-2$ 分子具有 $\Lambda$ 型结构(见图 1 和图 4), 含两个 对- $N, N$-二甲胺苯乙烯基分支. 两个分支之间的夹角 (即两个电荷转移轴之间的夹角) 为 $131^{\circ}$. NMe-2 分子 可以看作是由两个NMe-1 共用一个三嗪环的组合体. 晶体属于单斜晶系, $\mathrm{P} 21_{1}$ 空间群. 由于 $\beta=92.192(5)^{\circ}$, 非常接近于正交晶系. 晶胞中有 2 个分子, 关于 2 螺 旋轴对称等价.

\section{2 宏观非线性光学性质}

NMe-1, NEt-1 和NMe-2 这三个化合物在 $500 \mathrm{~nm}$
到近红外的波长范围没有吸收(见图 5), 在此范围它 们的近 100\% 的透过率可以满足 $1064 \mathrm{~nm}$ 倍频的要求. $\Lambda$ 型双支化合物 NMe-2 的吸收峰的强度 2 倍于相应的 单支化合物 NMe-1, 且 NMe-2 的吸收峰相对于NMe-1 有大约 $20 \mathrm{~nm}$ 的红移, 说明 NMe-2 的共轭程度大于 $\mathrm{NMe}-1$. 基于双能级模型 116$]$ 和上述光谱特点, $\mathrm{NMe}-2$ 分子应具有相对较强的二阶NLO效应.

化合物粉末晶样的 SHG 强度按 Kurtz 方法测 定 ${ }^{[17]}$, 以尿素粉末为参比, 为使结果有可比性, 所有 样品的颗粒度保持相同的范围. 皮秒脉冲 $\mathrm{Nd}$ : YAG激 光器的 $1064 \mathrm{~nm}$ 线为基频光源. NMe-1 没有 $\mathrm{SHG}$ 响应, 而NEt-1 和NMe-2 粉晶的SHG强度分别为尿素的 46.8 和 46.2 倍, 这与一些著名有机NLO晶体 (如NPP ${ }^{[4]}$, $\mathrm{BMC}^{[10]}$ )在同样波长范围下的SHG强度相当.

\section{3 微观非线性光学性质}

按照态加和(sum-over-state SOS)方法, 分子二阶 


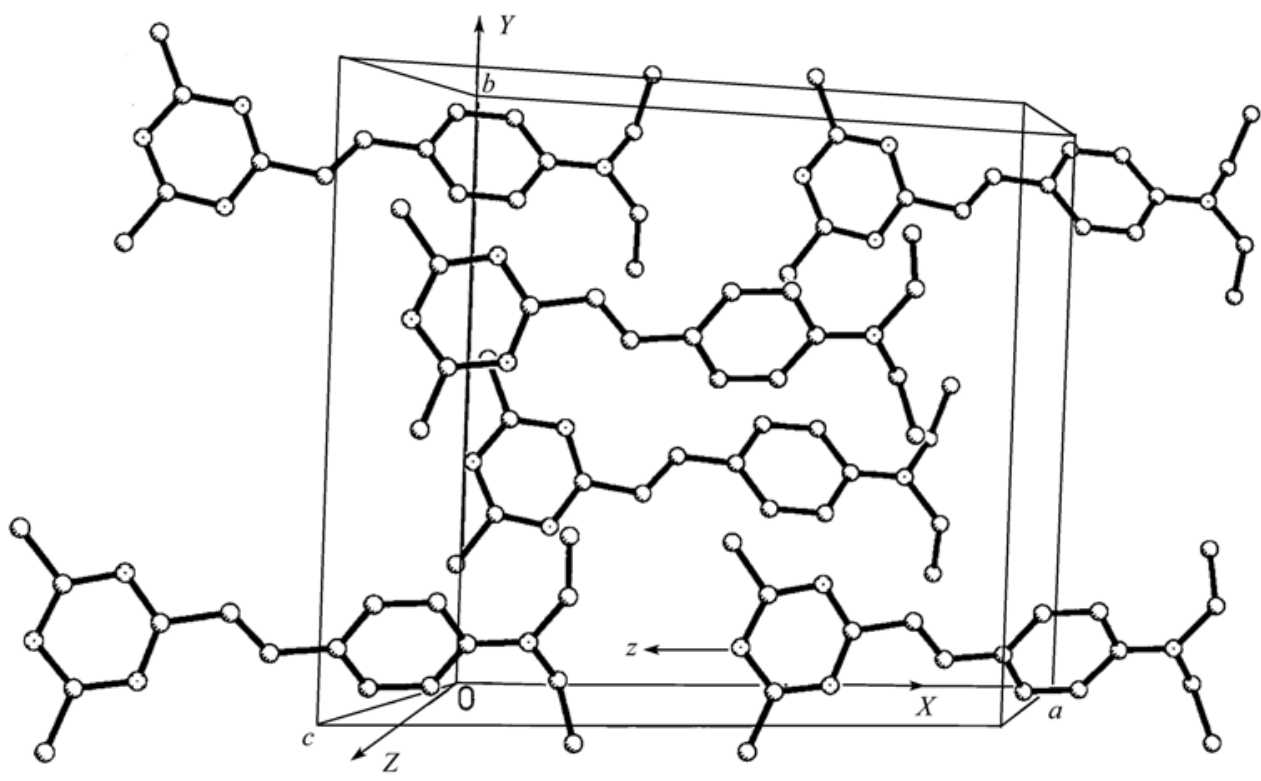

图 3 NEt-1 的晶体结构图

$z$ 轴为分子的电荷转移轴, 与 $a$ 轴平行. 晶体的宏观介电坐标轴 $X, Y$ 和 $Z$ 分别沿晶体学 $a, b$ 和 $c$ *轴

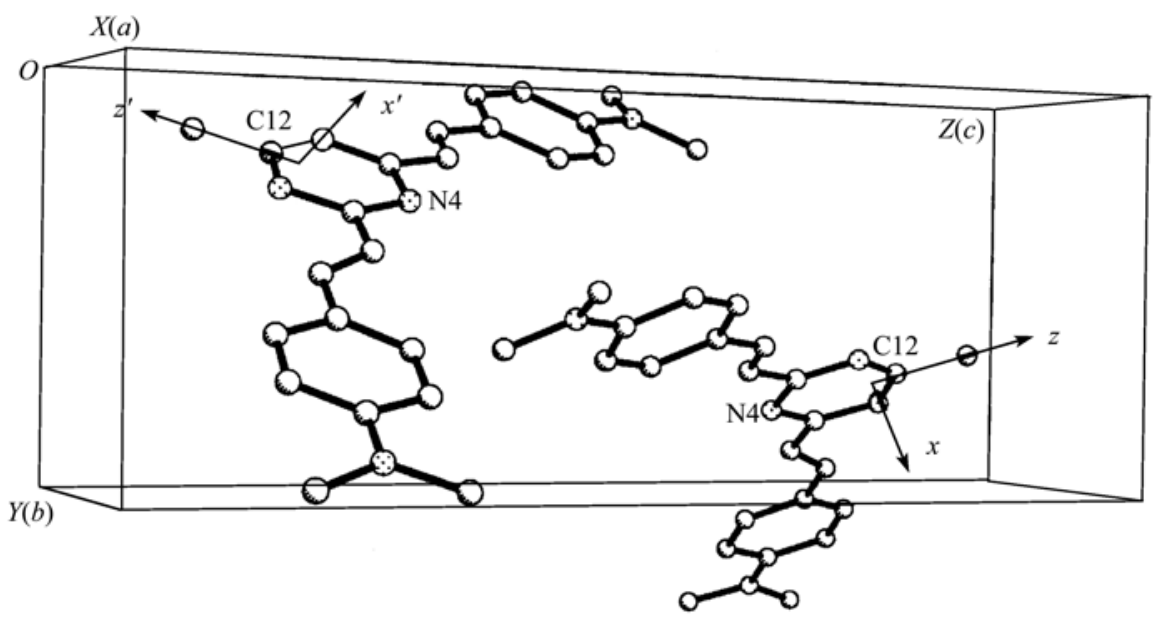

图 $4 \mathrm{NMe}-2$ 的晶体结构以及分子坐标和宏观介电坐标轴的定义

分子的 2 重轴(穿过 $\mathrm{C} 12$ 和 $\mathrm{N} 4$ 原子)为 $z$ 轴, $x$ 轴在分子的最小二乘平面内且垂直于 $z$ 轴. 晶体的宏观介电坐标轴 $X, Y, Z$ 可近似为晶体学坐标轴 $a, b, c$

极化率的笛卡尔分量 $\beta_{i j k}$ 表达为 ${ }^{[18]}$ :

$$
\begin{gathered}
\beta_{i j k}\left(-\omega_{\sigma}, \omega_{1}, \omega_{2}\right)=\frac{1}{\hbar^{2}} P\left(i, j, k ;-\omega_{\sigma}, \omega_{1}, \omega_{2}\right) \\
\sum_{l \neq 0} \sum_{m \neq 0} \frac{\left\langle 0\left|\mu_{i}\right| l\right\rangle\left\langle l\left|\bar{\mu}_{j}\right| m\right\rangle\left\langle m\left|\mu_{k}\right| 0\right\rangle}{\left(\omega_{0 l}-\omega_{\sigma}\right)\left(\omega_{0 m}-\omega_{2}\right)},
\end{gathered}
$$

其中, $i, j, k$ 对应于笛卡儿坐标 $x, y, z ; \quad \omega_{1}, \omega_{2}$ 是光场频
率, $\omega_{\sigma}=\omega_{1}+\omega_{2}$ 为极化分子的二阶响应频率，若 $\omega_{1}=\omega_{2}$ $=\omega$, 则有 $\omega_{\sigma}=2 \omega$, 对应于倍频过程; $P\left(i, j, k ;-\omega_{\sigma}\right.$, $\left.\omega_{1}, \omega_{2}\right)$ 是置换算符，定义为 $(i, j, k)$ 的任意一对置换与 $\left(-\omega_{\sigma}, \omega_{1}, \omega_{2}\right)$ 相应的一对置换同时进行; $l, m$ 表示激发 态, 0 表示基态; $\left\langle 0\left|\mu_{i}\right| l\right\rangle$ 表示分子沿 $i(=x, y, z)$ 轴方向 基态 $\langle 0|$ 和激发态 $\langle l|$ 之间的跃迁矩; $\left\langle l\left|\bar{\mu}_{j}\right| m\right\rangle=$ 
$\left\langle l\left|\mu_{j}\right| m\right\rangle-\left\langle 0\left|\mu_{j}\right| 0\right\rangle \delta_{l m} ; \omega_{0 l}=\left(E_{l}-E_{0}\right) / \hbar$ 为基态 $\langle 0|$ 到激发态 $\langle l|$ 的跃迁频率.

本文采用Gaussian 03 程序包 ${ }^{[19]}$ ，在CIS/6-31G水 平上计算了分子的基态和 20 个激发态的偶极矩、各 态之间的跃迁偶极矩和跃迁频率，利用上式计算分 子的二阶极化率. 计算中分子构型采用实测晶体结 构数据.

二阶极化率 $\beta$ 有 27 个分量, 由于固有对称性, 其 独立分量减少至 18 个. 在Kleinman角标交换全对称 性的假设下, 则 $\beta$ 的独立分量进一步减少为 10 个, 计 算结果很好的符合Kleinman假设. 计算中还发现下 标中含 $y$ 的 $\beta_{i j k}$ 都很小, 可以略去, 这与分子结构的平 面性一致. 表 2 给出基频光 $(\lambda=1064 \mathrm{~nm})$ 下各化合物 分子的各 $\beta$ 分量的计算结果.

由表 2 可见，三种分子的激发态偶极矩显著大于 基态偶极矩, 因而属于电荷转移型分子, 这种电荷转 移的性质得到溶致变色实验结果的支持. NMe-1 和 NEt-1 显然是一维电荷转移分子, 沿电荷转移轴 $z$ 方 向的 $\beta_{z z z}$ 分量构成 $\beta$ 的主要分量. 应该指出，二阶极化 率的计算结果与态求和时所取的激发态的数目 $n$ 有关, 然而, 对NEt-1 的 $\beta_{z z z}$ 分量的计算结果表明, $n<10$ 时结 果随 $n$ 的变化较大, $n=12$ 时趋向饱和收敛, 此后一 直计算到 $n=30, \beta_{z z z}$ 基本不变, 因而我们在所有计算 中取 $n=20$.

参见图 3 及 2, NEt-1 的分子结构与 NMe-1 类似, 但三嗪环与苯环的平面间的夹角为 $27.7^{\circ}$, 分子的平 面性相对较差. 由于 $\mathrm{NMe}-1$ 的共轭性相对较好, 因而 其 $\beta$ 值大于 NEt- 1 的 $\beta$ 值(这里的 $\beta$ 值指 $\beta$ 的范数 $\|\beta\|$ ).

参见图 4, 双支分子 $\mathrm{NMe}-2$ 具有很好的平面性, 两个分支的最小二乘平面之间的夹角仅为 $5.9^{\circ}$. 如果 忽略这一小角, 分子具有 $C_{2 v}$ 对称性, 具有二维对称
电荷转移特征. 由于两个电荷转移轴间的夹角 $\left(131^{\circ}\right)$ 相当大，可以预期 NMe-2 的基态偶极矩和激发态偶 极矩都不会很大，计算结果也是这样(见表 2). NMe-2 较大的 $\beta$ 值对应于其较大的共轭程度和对称电荷转 移模式, 由此导致较大的跃迁矩和较小的跃迁能(参 见图 5). 二维电荷转移分子 $\mathrm{NMe}-2$ 的 $\beta$ 张量的另一 特点是，起主导作用的不是对角张量元，而是非对角 元 $\beta_{z x x}$.

\section{4 宏观与微观非线性光学性质的关联}

有机晶体中，分子间的范德华力远弱于分子内 的化学键，可以采用忽略分子间的相互作用的取向 气体模型 (oriented gas model). 在此模型下, 晶体的 二阶极化率 $\chi_{I J K}^{(2)}$ 与下述 $b_{I J K}$ 成正比 ${ }^{[1]}$ :

$$
b_{I J K}=\frac{1}{N} \sum_{i j k} \sum_{s=1}^{N} \cos \theta_{I i}^{(s)} \cos \theta_{J j}^{(s)} \cos \theta_{K k}^{(s)} \beta_{i j k},
$$

其中, $N$ 是晶胞内的对称等效分子数. $\theta_{l i}$ 为介电坐标轴 $I$ 与分子坐标轴 $i$ 之间的夹角. 显然 $b_{I J K}$ 是介电坐标 系下分子二阶极化率 $\beta$ 的平均值，即每个分子对

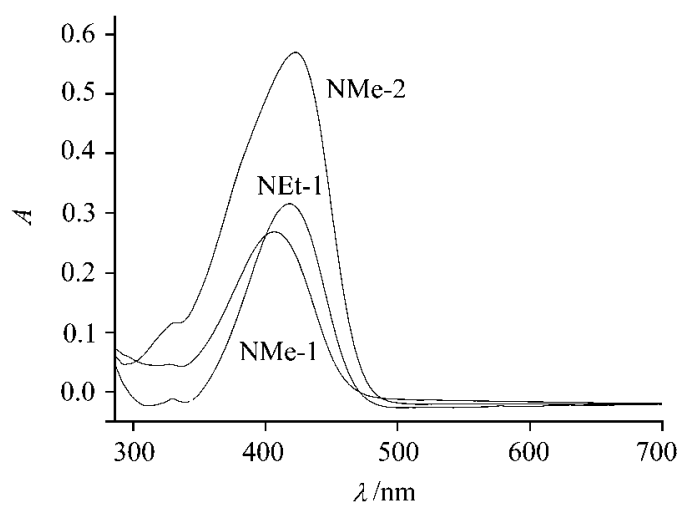

图 5 化合物 NMe-1, NEt-1 和NMe-2 在氯仿溶剂中的吸收 谱 $\left(C=1.0 \times 10^{-5} \mathrm{~mol} \cdot \mathrm{L}^{-1}\right)$

表 2 分子的基态偶极矩 $\mu_{0}(\mathrm{D})$ 第 1 激发态偶极矩 $\mu_{1}(\mathrm{D})$, 二阶极化率 $\beta\left(10^{-30} \mathrm{esu}\right)$ 的计算值 ${ }^{\mathrm{a})}$

\begin{tabular}{ccccccccc}
\hline & $\mu_{0}$ & $\mu_{1}$ & $\beta_{z z z}$ & $\beta_{z x x}$ & $\beta_{x x x}$ & $\beta_{x z z}$ & $\|\beta\|^{\text {b) }}$ & SHG 强度 \\
\hline NMe-1 & 5.113 & 11.386 & -87.18 & 3.6525 & -0.6221 & -4.799 & 87.9 & 0 \\
NEt-1 & 4.276 & 11.18 & -71.08 & 1.136 & -0.4657 & 1.488 & 79.9 & $46.8 \mathrm{U}$ \\
NMe-2 & 3.372 & 6.314 & -6.646 & -60.896 & -3.086 & -0.8862 & 105.9 & $46.2 \mathrm{U}$ \\
\hline
\end{tabular}

a) $\beta$ 中的色散变量固定为 $\lambda=1064 \mathrm{~nm}$.

b) $\beta$ 的范数 $\|\beta\|$ 按下式定义: $\|\beta\|^{2}=\sum_{i j k} \beta_{i j k}^{2}$, 根据此式计算时对所有 27 个分量求和 
宏观晶体二阶极化率指定分量的贡献. (2)式即忽略 分子间的相互作用情况下, 宏观与微观非线性光学 系数的关系式，它表明晶体的二阶非线性响应取决 于其分子的二阶极化率 $\beta$ 以及分子的堆积方式.

$\mathrm{NMe}-1$ 虽然具有较大的 $\beta$ 值, 但由于晶体的中心 对称性, 无 SHG 效应.

NEt-1 晶体属于极性点群 $m$, Kleinman 近似下, 有 6 个独立的 $\chi^{(2)}$ 系数, 由于分子电荷转移轴 $(z$ 轴)在 晶体宏观介电坐标系 XYZ(见图 3)下的方向余弦为 $[-0.9999,-0.0071,-0.0049]$, 由于晶体的对称特性, 所有分子的电荷转移轴的方向完全一致, 并与晶体 学 $a$ 轴平行. 在一维分子的模型下, 可以得到非常简 洁的下述结果:

$$
\begin{gathered}
b_{X Y Y}=b_{X Z Z}=b_{X X Z}=b_{Y Y Z}=b_{Z Z Z}=0, \\
b_{X X X}=\cos ^{3} \theta_{X Z} \beta_{z Z z}=-\beta_{z Z z}=71.08 \times 10^{-30} \mathrm{esu},
\end{gathered}
$$

这种自然最大极化的优化堆积在有机NLO晶体中很 少见，据我们所知，目前只有三例有机NLO晶体采取 这种堆积方式 ${ }^{[7,8,10]}$. NEt-1 晶体的宏观极化强度达到 极大, 而NMe-1 晶体的宏观极化强度为零(中心对称), 造成晶体结构巨大差别的原因当然是分子结构. NEt-1 与NMe-1 的分子很相似, 但有两点不同：1) NEt-1 的基态偶极矩较小 (见表 2), 2) NEt-1 的电子给 体端和受体端的宽度几乎相同(见图 3), 此外乙基还 具有一定的柔性. 上述因素可能有利于分子的电荷 转移轴趋于同向平行排列.

$\mathrm{NMe}-2$ 晶体属于点群 2 , 有 4 个独立的 $\chi^{(2)}$ 系数: 将具体的晶体学数据代入表达式(2),得:

$$
\begin{aligned}
& b_{X Y Z}=-0.1162 \beta_{z z z}-0.0344 \beta_{z x x}=2.867 \times 10^{-30} \mathrm{esu}, \\
& b_{X X Y}=-0.0623 \beta_{z z z}-0.4424 \beta_{z x x}=27.35 \times 10^{-30} \mathrm{esu}, \\
& b_{Y Y Y}=-0.0293 \beta_{z z z}-0.1490 \beta_{z x x}=9.268 \times 10^{-30} \mathrm{esu}, \\
& b_{Y Z Z}=-0.2165 \beta_{z z z}+0.2696 \beta_{z x x}=-14.98 \times 10^{-30} \mathrm{esu},
\end{aligned}
$$

$\mathrm{NMe}-2$ 晶体未达到最佳分子堆积. 由表 2 可见, NEt-1 的微观 $\beta$ 值虽然小于 $\mathrm{NMe}-2$, 但由于最佳堆积, NEt-1 晶体的宏观 SHG 强度超过了 NMe-2 晶体.

\section{3 小结}

有机晶体的宏观 NLO 响应取决于其分子的 NLO
性质以及分子的堆积方式. 本文在合成工作和结构 测定工作的基础上，综合考察了一组均三嗪衍生物 的分子结构与其微观二阶 NLO 性质的关系，以及由 分子结构决定的晶体结构与宏观二阶 NLO 性质的关 系. 验证了如下分子设计思想: (1) 大的共轭范围和 二维电荷转移模式有利于提高分子二阶极化率; (2) 基态下分子的强极性并不重要(强极性反而导致中心 对称的堆积), 重要的是激发态下电荷转移导致的极 性增强. 在新材料探索方面, 本文得到了两个具有很 强 SHG 效应的化合物及其晶体, 其中 NEt-1 晶体中 分子电荷转移轴同向平行排列, 实现了最佳堆积.

\section{参 考 文 献}

1 Chemla D S, Zyss J. Nonlinear Optical Properties of Organic Molecules and Crystals. Vol I, Orlando: Academic Press, 1987

2 叶 成, Zyss J. 分子非线性光学的理论与实践. 北京: 化学工业 出版社. 1996

3 Verbiest T, Houbrechts S, Kauranen M, et al. Second-order nonlinear optical materials: recent advances in chromophore design. J Mater Chem, 1997, 7(11): 2175 2189[DOI]

4 Zyss J, Nicoud J F, Coquillay M. Chirality and hydrogen bonding in molecular crystals for phase-matched second-harmonic generation: N-(4-nitrophenyl)-(L)-prolinol (NPP). J Chem Phys, 1984, 81(9): 4160 4167 [DOI]

5 Tam W, Guerin B, Calabrese J C, et al. 3-Methyl-4-methoxy-4'nitrostilbene (MMONS): Crystal structure of a highly efficient material for second-harmonic generation. Chem Phys Lett, 1989, 154(2): 93 96[DOI]

6 Luo J D, Hua J L, Qin J G, et al. The design of second-order nonlinear optical chromophores exhibiting blue-shifted absorption and large nonlinearities: The role of the combined conjugation bridge. Chem Commun, 2001, 171 2

7 Ohkita M, Suzuki T, Nakatani K, et al. Crystal engineering using very short and linear $\mathrm{C}(\mathrm{sp})-\mathrm{H}$... N hydrogen bonds: Formation of head-to-tail straight tapes and their assembly into nonlinear optical polar crystals. Chem Commun, 2001, 1454 1455

8 Zyss J, Ledoux I, Bertault M, et al. Dimethylaminocyanobiphenyl (DMACB): A new optimized molecular crystal for quadratic nonlinear optics in the visible. Chem Phys, 1991, 150: 125 135[DOI]

9 Marder S R, Perry J W, Schaefer W P. Synthesis of organic salts with large second-order optical nonlinearities. Science, 1989, 245: 626 628

10 Li Z D, Huang L R, Su G B, et al. Crystal structure of 4bromo-4'-methoxyl-chalcone. J Struct Chem, 1992, 11(1): 1 4

11 Zyss J, Chemla D S, Nicoud J-F. Demonstration of efficient non- 
linear optical crystals with vanishing molecular dipole moment: Second-harmonic generation in 3-methyl-4-nitropyridine- 1-oxide. J Chem Phys, 1981, 74(9): 4800 4811[DOI]

12 Fang Q, Jiang M H, Qu Z, et al. Nonlinear optical properties of DMIT derivatives. J Mater Chem, 1994, 4(7): 1041 1045[DOI]

13 Zyss J. Octupolar organic systems in quadratic nonlinear optics: molecules and materials. Nonlinear optics, 1991, 1: 3 18

14 Cho B R, Park S B, Lee S J, et al. 1,3,5-tricyano-2,4, 6-tris(vinyl) benzene derivatives with large second-order nonlinear optical properties. J Am Chem Soc, 2001, 123: 6421 6422[DOI]

15 Cui Y Z, Fang Q, Lei H, et al. Syntheses, structure and second-order nonlinear optical properties of octupolar compounds: 2,4,6- tri-substituted $s$-triazine. Chem Phys Lett, 2003, 377: 507 $511[\mathrm{DOI}]$

16 Oudar J L, Chemla D S. Hyperpolarizabilities of the nitroanilines and their relations to the excited state dipole moment. J Chem Phys, 1977, 66(6): 2664 2668

17 Kurtz S K, Perry T T. A powder technique for the evaluation of nonlinear optical materials. J Appl Phys, 1968, 39 (8): 3798 3813

18 Willetts A, Rice J E, Burland D M, et al. Problems in the comparison of theoretical and experimental hyperpolarizabilities. J Chem Phys, 1992, 97(10): 7590 7599[DOI]

19 Frisch M J, Trucks G W, Schlegel H B, et al. Gaussian 03, Revision B.5. Pittsburgh PA: Gaussian Inc, 2003 